\title{
PERIPHERAL ARTERIAL DISEASES
}

\author{
By Saul S. Samuels, A.M., M.D. \\ New York, N.Y.
}

\section{Peripheral Arterial Diseases}

This new specialty has evolved within the past twenty years into a separate and distinct branch of medicine and surgery. In its infancy it was relegated to the internist who had some special interest in this work, just as in the early days of urology an acquaintance with venereal diseases was sufficient for the designation of specialist in this field.

\section{General Considerations}

The peripheral arterial diseases may be separated into two definite groups. The first and more important is that of the changes in the arterial walls. These two groups may be again subdivided so that in the organic field we recognise two major varieties of organic arterial disease in the extremities. The most common of these, and by far the most important from the standpoint of frequency of occurrence, is arteriosclerosis. At this point it might be well to differentiate the term arteriosclerosis from the disease process known as arteriosclerosis obliterans. The practical significance of this distinction lies in the fact that arteriosclerosis, that is to say, definite structural changes in the walls of the arteries may be present to a great degree without in any way interfering with the arterial onflow. In other words, the mere presence of arteriosclerosis of the peripheral arteries as shown by roentgen films does not in itself indicate arterial insufficiency in that particular individual. When, however, the element of obstruction is added to arteriosclerosis, induced either by progressive narrowing of the lumen of the artery or by encroachment on its blood carrying capacity by the formation of thrombi on mural plaques, insufficiency of the arterial flow may then be expected. In such a case the proper terminology is arteriosclerosis obliterans. The frequent association of an arteriosclerotic process with diabetes mellitus accentuates the importance of this disease in the field of peripheral vascular diseases. Whether or no the presence of diabetes intensifies the arteriosclerotic process, particularly in the uncontrolled diabetic patient, is still open to question. In any event the combination of the two adds considerable difficulty to the treatment of gangrene of the extremities.

The other important organic arterial disease is thromboangiitis obliterans, commonly known as Buerger's Disease. This will be described in greater detail in the following pages. Aside from arteriosclerosis and thromboangiitis obliterans, there are other organic diseases of the peripheral arteries that might be mentioned, but clinical evidence of which is difficult to determine. They are encountered so infrequently that it hardly seems important to devote much time to them. Among these may be mentioned syphilitic arteritis, tuberculosis of the peripheral arteries and certain non-specific forms of peripheral arteritis, which have as yet been incompletely studied.

In the field of functional disturbances of the peripheral arterial system, we consider two main sub-groups. The first, and more important of these is Raynaud's Disease. This is a disturbance of the control mechanism of the peripheral arterioles which causes frequent attacks of vasospasm in the extremities and in the various acral parts of the body, such as the tip of the nose, tongue and ears. It is frequently associated with a peculiar trophic disturbance of the skin, hands, arms, face and chest. This is a distinct variety of scleroderma. Dermatologists seem to distinguish this form of scleroderma from that which is not encountered in Raynaud's Disease and which appears to be preceded by an inflammatory reaction in the dermal tissues. Another frequent accompaniment of Raynaud's Disease is a peculiar variety of rheumatoid arthritis, involving usually the small joints of the hands and feet and in some instances the larger joints of the body.

The other functional derangement of the peripheral arterial system is known as erythromelalgia or Weir Mitchell's Disease. This interesting but extremely rare disease will be mentioned here merely for the sake of completeness, but from a practical standpoint it is so seldom encountered as to require comparatively little consideration. Its main features are a paralysis of the vasomotor mechanism to such an extent that an almost constant vasodilatation is obtained in all four extremities. In some instances, however, the disturbance may be confined to a single limb. I have seen such a case resulting from severe trauma to an upper extremity. According to Brown of the Mayo Clinic, the diagnostic features of erythromelalgia are severe burning pain in the affected extremities which symptoms are relieved only by immersion of the affected parts in ice-cold water. He stresses the importance of this means of obtaining relief as an essential factor in arriving at a diagnosis. It is important to recognise this fact because frequently both in practice and in the literature of peripheral vascular diseases the error has been made of diagnosing erythromelalgia merely on the basis of redness and perhaps some swelling with burning pain of one or more extremities. It must not be forgotten that the condition known as rubor, parti- 


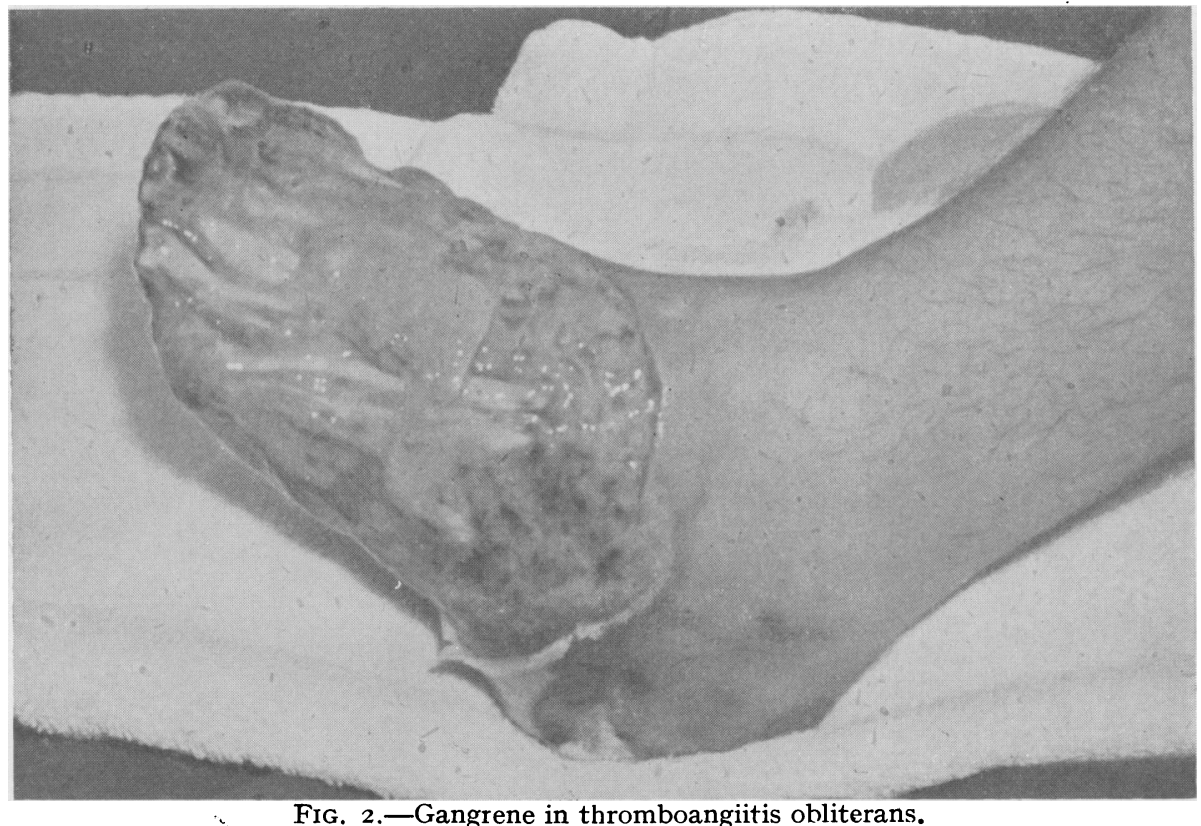

FIG. I.-Samuels' Test for plantar ischemia. 


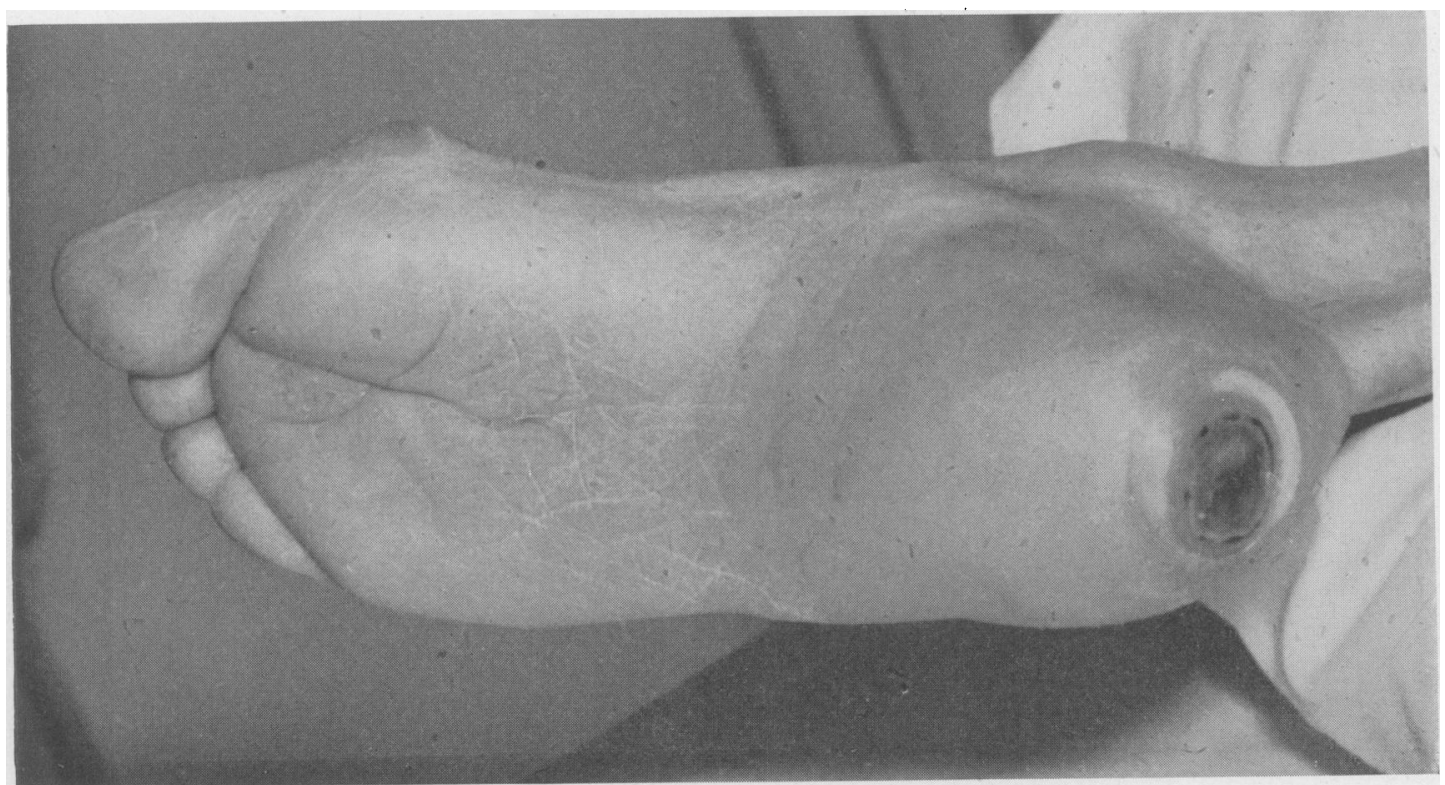

FIG. 4.-Diabetic gangrene of heel.

FIG. 5.-Same case healed. 
cularly in the lower extremities, is frequently present in long-standing cases of arteriosclerosis obliterans or in thromboangiitis obliterans and to the inexperienced observer the appearance of this one stage, namely rubor, may induce an erroneous diagnosis of erythromelalgia.

Here I wish to stress the fact that in recent years there has been a great tendency to overemphasise the importance of vasomotor change, particularly vasospasm, in the organic arterial diseases. Unfortunately, therapy of the organic diseases has been directed in many instances to the elimination of vasospasm. The fallacy of this concept lies in the fact that the vasomotor disturbances associated with the organic arterial diseases are for the most part confined to the very early stages of these diseases and are comparatively infrequent in the later stages where the organic element is a predominant feature. In accordance with this misconception, too many operative procedures, particularly sympathectomy, have been advocated and been performed unnecessarily in cases where simpler methods of treatment were indicated.

\section{Symptomatology}

At the outset it is important to know that the symptoms of arterial insufficiency in the extremities are extremely variable and may be absent entirely in the presence of advanced occlusive disease, or they may simulate many other common conditions. For these reasons, it is important to lay more stress on the objective findings elicited by a careful physical examination, rather than on the patient's statement of symptoms. Failure to recognise this point has resulted in numerous incorrect diagnoses on the part of excellent practitioners and specialists, who apparently rely too much upon the patient's story. A familiar story is that of the patient with symptoms of pain radiating down the back of the thigh originating in the buttock, which is so often incorrectly ascribed to sciatica. As a matter of fact, this is a fairly common symptom of arterial insufficiency of the lower extremities, particularly in the early stages of arteriosclerosis obliterans. Other misleading symptoms are pains in various parts of the feet which simulate the symptoms of pes planus. Again, particularly in arteriosclerosis, the symptoms may resemble almost completely those of arthritis of the knees and ankles. The common association of osteoarthritis with peripheral arteriosclerosis obliterans has been observed and reported by Steinbrocker and myself. It behoves one, therefore, in studying middle aged or elderly patients with arthritic symptoms in the lower extremities, to carry out a complete examination of the peripheral arterial system before passing final judgment as to the cause of the symptoms.

Just as in coronary artery disease where the general clinical picture is frequently called angina pectoris, so in the field of arterial insufficiency of the lower extremities one groups all the symptoms together in the category of intermittent claudication. In its classic form this syndrome consists of a feeling of fatigue or actual cramp in the calf of the affected leg induced by walking, particularly fast walking or climbing a flight of steps. Thus, after a variable distance, often depending upon the severity of the arterial disease, the patient is forced to stop because of disabling symptoms. After a rest period of a moment or so, the patient is able to proceed for a comparatively short distance after which the disabling symptom reappears. This classic form of intermittent claudication does not, however, always manifest itself in every case. As mentioned previously, it may resemble a form of sciatic pain or it may appear as a tightening of the small muscles of the foot, inducing a cramp-like seizure which does not allow the patient to proceed in walking, or there may be numbness of the toes or of the entire foot or leg. Paresthesias of various kinds can also be brought to light after walking a short distance and may be the only sign called to the patient's notice that something un usual is occurring in the feet. Any of these symp toms may be accompanied by subjective coldness of either the toes or the entire foot of the affectee extremity. This is particularly true in those cases where there has been a fairly recent interference with arterial flow such as in a thrombosis of a main vessel. Arriving at a diagnosis merely on symptoms alone has, of course, led to many strange therapeutic procedures. A prescription of arch supports, of specially constructed shoes, of various intricate strappings of the feet and legs have all been encountered. It is well to stress again at this point the fact that symptoms may be extremely disabling in the mildest form of organic disease, while in some cases patients with extreme insufficiency in an extremity may insist that all their trouble lies in the opposite leg in which the physician finds the least evidence of arterial disease. We thus come to the most important part of the study for purposes of diagnosis of these cases, namely, the various diagnostic procedures at our disposal.

\section{Diagnostic Procedures}

Before outlining the best methods of arriving at a diagnosis of peripheral arterial obstruction, it will be well to discuss the classical procedure which has been taught as the standard in text-books and by many teachers. I refer to the procedure of palpating the dorsalis pedis pulse. It has been 
taught that the absence of this pulse by palpation is sufficient indication for the diagnosis of peripheral arterial disease. And conversely, that the presence of this pulse indicates the absence of arterial disease. Nothing could be further from the truth. If one assumes that the dorsalis pedis artery can be felt to pulsate at its usual prominent point on the dorsum of the foot, one is not thereby told the condition of the distal distribution of the dorsalis pedis artery and its branches. In other words, incipient arterial disease may be present in the extremity even though the dorsalis pedis artery pulsates. The same can be said of the posterior tibial artery which can be felt in only a small portion of its extent behind and below the internal malleolus. If the examining physician detects pulsation of this portion of the artery, there is given no information on the status of the posterior tibial artery distal to its point of palpation, nor does one have any information concerning the condition of the plantar arterial system.

A more accurate and more sensitive approach to the question of diagnosis must therefore be used. In the advanced case of organic disease mere inspection of the foot will, in almost every instance, reveal the presence of pallor or ischemia in varying degree, depending upon the extent of the arterial obstruction. In other words, a badly involved leg will show extreme pallor of the foot, either of the plantar surface or of the foot as a whole if the patient is in the reclining position. In some cases this ischemia may be so severe as to resemble the foot of a cadaver. In the slightly involved cases ischemia is not easily seen except by special methods. Elevation of the feet above the level of the heart, obtained by having the patient flat with legs elevated to an angle of at least $45^{\circ}$ will in the moderately involved case produce ischemia or blanching of the plantar surface of the affected extremity. Fortunately in the organic arterial diseases the condition is usually asymmetric and relative colour changes are easily discernible. I must stress the importance of good even light falling upon the plantar surfaces of the feet as they are elevated. In the very early cases elevation alone may be insufficient to bring out ischemia. Therefore a reinforcement of the test is necessary. This consists in a pumping action of the leg, produced by having the patient flex the feet very rapidly, using the ankle joints as pivots and not the toes alone, for a few moments. During this time excess blood of both extremities will be pumped by the calf muscles and the action of gravity out of the plantar surface of the feet and a distinct and unmistakable plantar ischemia will be elicited in the involved extremities. This colour change is apparent even in the earliest cases of arterial disease and in those where the dorsalis pedis and posterior tibial pulses may still be palpated. It is thus seen how important this simple test for plantap ischemia is for arriving at a diagnosis of peripheras arterial obstruction. Ischemia cannot be elicited in the pure vasospastic diseases such as Raynaud's. Disease. For here the ischemia is pure vasospasm $\overrightarrow{\vec{F}}$ and can be induced only by those causes whicto produce it as a form of disease, namely exposure tof cold or emotional disturbance. Thus in the sign otn. plantar ischemia we have pathognomonic evidence् of the existence of organic arterial insufficiency inf an extremity.

Relative coldness of the affected extremity is to be expected in the ischemic foot. This is a con $=$ firmatory sign and may be recognised easily by manual palpation of the feet in corresponding areaso Delicate electrical thermometers and other simila 5 instruments of precision are unnecessary for ordi nary examination. They do have a place in re $N$ search or advanced study of peripheral arteria phenomena, but for routine examination simple manual palpation is sufficient. Thus there are two simple procedures at one's disposal for arriving af a diagnosis. It is unfortunate that these methods have been slighted in the past and it is to be hopedo that they will soon supplant the inadequate an misleading procedure of pulse palpation.

As in the study of hypertension, one is not satis= fied with mere qualitative diagnosis. One prefors mechanical proof of the existence of either hypers? tension or of organic arterial obstruction. Wiat? the sphygmomanometer is to blood pressure study so is the oscillometer to the consideration of perio pheral arterial disease. Rather than go into historical discussion of this interesting instrument I will refer merely to two types in general use $\frac{7}{5}$ namely the visual type, corresponding to the ordinary blood pressure apparatus and the recordo ing type of oscillometer. Of the two the latter is by far the better, both from the standpoint of accus racy and of permanence of record. In other words an accurate impressional tracing of pulsation cari be obtained, while in the visual type one must. follow the travels of the indicating needle over $ळ$ dial and must contemplate the extent or amplitude of each pulsation. The oscillometer measures the pulsation of the main arteries under pulsation. I thus represents the sum total of pulsation of all o. the main vessels at any particular level. Unfor tunately it does not measure the collateral circus lation which is usually made up, for the most parts of a network of tiny vessels which apparently d\&్ర not pulsate, but form a continuous flow. Clinicab evidence of collateral circulation must be obtained by other means. For instance, increase in warmtlई of the extremity, improved colour and, of course ${ }_{?}^{\infty}$ the healing of gangrenous ulcers are all indications of the improved collateral circulation which cannot 


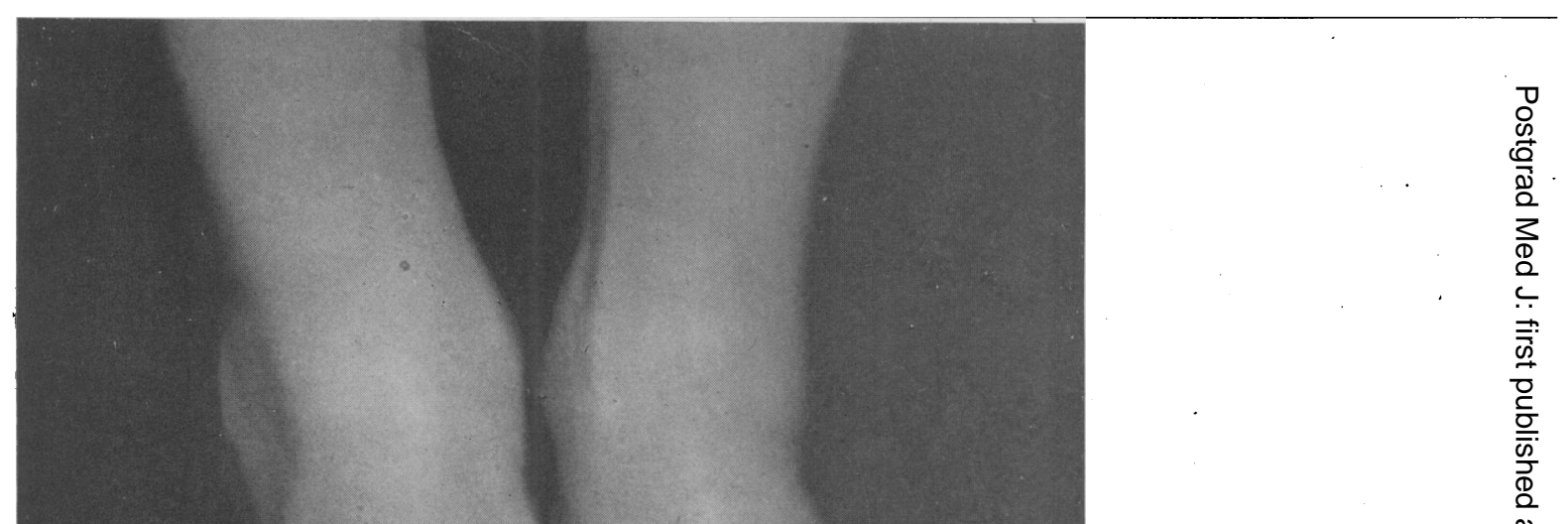

FIG. 9.-Bilateral diabetic gangrene healed.

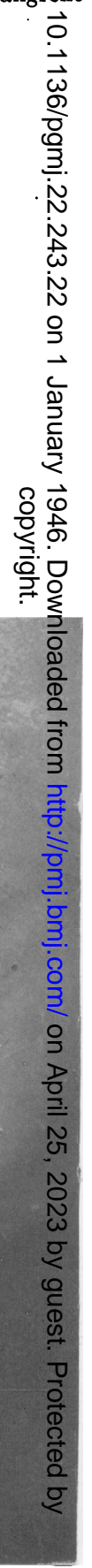


PERIPHERAL ARTERIAL DISEASES. SAul S. SAmuels, A.M., M.D.

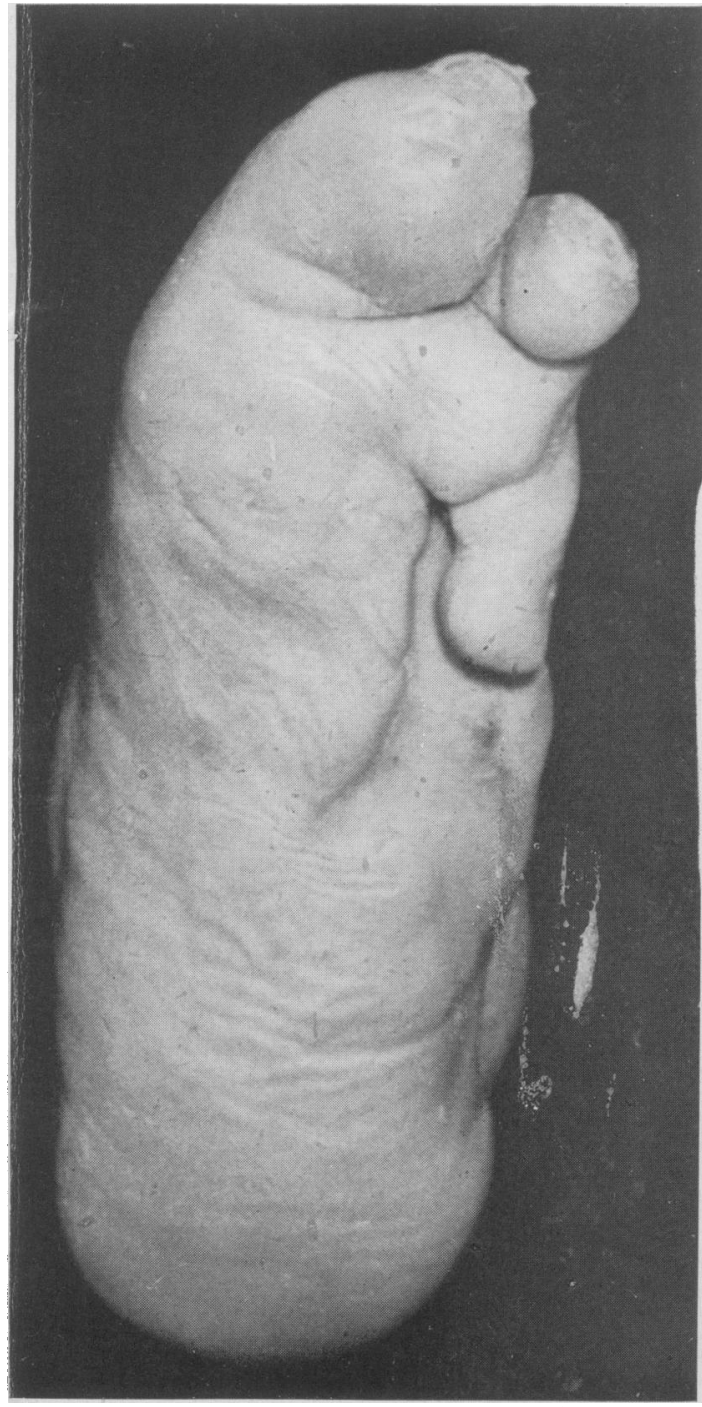

FIG. I I.-Same case, plantar view.

FIG. 12.-Healed arteriosclerotic gangrene. 
be measured by the oscillometer. The diagnostic importance of the oscillometer lies in the fact that one is able to measure quantitatively the relative extent of arterial pulsation in the extremity and as there is usually an asymmetric involvement of the arterial circulation in organic diseases, the relative values are of considerable importance. Thus there are no normal figures for oscillometric readings since the gradations of the machine are purely arbitrary. However, as a general rule, one may say that the normal range of oscillometric readings at the ankle level is within two to ten divisions of the scale. As the cuff is applied proximally on the limb the normal range naturally increases so that at the popliteal region one may expect a normal range of from five to twenty divisions and proportionately higher values on the thigh. The same applies to readings taken on the upper extremities. They are less in extent at the periphery of the extremity and increase as one approaches the body. It is obvious, therefore, that relative readings on opposite limbs at the same level are more accurate and of greater importance than the actual figures themselves.

A vital value of the oscillometer lies in its ability to furnish information concerning prognosis. It has been observed over a long period that those cases wherein the oscillometer needle does not pulsate at the ankle level have a relatively poor prognosis, particularly in regard to conservative treatment. This is readily understood when it is known that the oscillometer records pulsation of the large vessels only and if there is no pulsation, one must conclude that the large vessels are completely blocked and that one may therefore assume that the collateral circulation stemming from these blocked vessels is correspondingly poor, hence the chances for success of conservative therapy are small. On the other hand, the presence of a pulsation as revealed by the oscillometer at the ankle level, no matter how small, should give one encouragement in the prosecution of conservative treatment in spite of all other apparent difficulties.

A word here concerning the use of various procedures and tests for the determination of vasospasm in the affected extremity. It must first of all be understood that vasospasm is of importance only in the very early stages of organic arterial disease and is usually completely absent in the advanced stages. Furthermore, it must also be recognised that it plays only a secondary part in the clinical picture of these diseases and when present, as in the early stages, is easily overcome by simple therapeutic procedures. Unfortunately, its influence has been over-exaggerated, particularly in the treatment of thromboangiitis obliterans and many tests and diagnostic methods have been advocated to determine its presence or absence.
Unfortunately many of these procedures involve्ष the use of nerve blocks of varying degree, of the administration of general anaesthetics and some times of the use of spinal anesthesia. Since the information obtained from these tests is of comparatively little value, and as the effect of these $\vec{E}$. procedures may be disastrous, it is not recomb mended that they be carried out. A detaile description of these various procedures for the. determination of vasospasm may be obtained fror standard text-books and will not be described if this paper.

Roentgen examination of the extremities ma $\vec{\Phi}$ show calcification of the tibial arteries. This is merely confirmatory evidence of an arteriosclerotifes process and is in no sense to be interpreted as diagnostic test of arterial insufficiency. In othe words, extensive arteriosclerosis with calcificatio of the vessel walls may be present and yet there may be no interference with arterial circulation When the element of narrowing of the arterial lumen is involved, either by thickening of the arterial wall or by thrombotic occlusion in the vessels, one may suspect arterial insufficiency which may be determined by the clinical testog enumerated previously.

\section{Etiology}

The essential difference between thromboangiitis obliterans and arteriosclerosis obliterans in the fact that the former definitely is an inflams matory disease of the arteries and veins, whilo arteriosclerosis is a structural modification of the arteries probably induced by metabolic disturlis ances. At no time in the course of this disease there evidence, either clinically or pathologicallys of inflammatory reaction. The use of the worg angiitis in Buerger's Disease implies at once the involvement of both arteries and veins in the inflammatory process in contrast to arterioscleros where the changes are confined to the arteries alone The inflammatory nature of Buerger's Disease immediately suggests the possibility of some agenf as the exciting cause of the malady. Buerger was of the opinion that the noxious agent lay in the inflamed vessel and could be easily transmitted from vessel to vessel or from person to perso Other investigators believed that an unidentifiad streptococcus was the cause of the disease. How? ever, a great drawback to the possibility of microbls involvement lay in the fact that the disease essentially one of males and very rarely exists females. The tendency to racial selection is also unexplainable from the standpoint of a germ disease.

I have for a long time been of the opinion that some infectious agent is at work in thromboangiitas 
obliterans, basing my opinion upon the acute inflammatory process so often found in both arteries and veins and in the presence of large giant cells characteristic of the disease. This giant cell, corresponding to the foreign body giant cell of non-specific infections, prompted me to the conclusion that we are undoubtedly dealing with either a protozoon or a member of the fungus group. Since various strains of fungi are present in the normal foot, and in many instances call forth the disease known as "athlete's foot" and since this latter is so predominant in the population, while Buerger's Disease is comparatively rare, it occurred to me that we were perhaps dealing with a specific as yet unidentified strain of fungus as the actual cause of the disease. It is interesting to note that according to dermatologists, fungous or mycotic infections of the feet are more frequent in males than in females. Thompson has recently made an exhaustive study of fungus infections of the feet and has noted that there are more cases of fungus infection of the feet in thromboangiitis obliterans than in the normal population. I thoroughly agree with Thompson and other investigators in this field but feel that a specific organism must be isolated before definite conclusions can be drawn. Isolation of the suspected fungus from an area of acute migrating phlebitis will undoubtedly go far towards confirming this theory.

As for the etiologic studies of arteriosclerosis, one must read the monograph by Winternitz to appreciate his theory which may be correct. Other explanations are for the most part theoretical and have little practical value. The influence of uncontrolled diabetes mellitus must not be underestimated in hastening and accentuating an underlying arteriosclerotic process.

Whether or not syphilitic arteritis of the legs exists as a specific disease is open to question. Final judgment must rest upon actual pathologic examination of amputated legs or excised vessels. Diagnosis from a clinical basis alone is inaccurate since the mere presence of a positive Wassermann or Kahn Test may be purely coincidental and does not necessarily indicate that a syphilitic process is present in the arteries of the extremities. I have encountered numerous instances of both Buerger's Disease and arteriosclerosis obliterans with positive Wassermann reactions, but in no case have I been convinced that I was dealing primarily with syphilitic disease.

\section{Treatment of Peripheral Arterial Disease}

In this presentation I shall attempt to describe the general principles upon which the details of treatment are to be based. The conclusions that I have drawn are the result of over twenty years' experience in both private and hospital practice in the treatment of ambulatory and bedridden cases of peripheral arterial disease. While some of my methods and ideas may be contradictory to those generally taught and held in the profession generally, the results obtained undoubtedly speak for themselves.

The first principle of importance is the realisation by the physician and patient that the vasoconstricting action of smoking is definitely harmful in all varieties and in all stages of peripheral arterial disease. While this fact has been known empirically for many years, it is only lately that carefully controlled experiments have proven the correctness of this theory. Experiments by many observers using delicately adjusted electrical thermometers attached to the finger tips and toes have shown that while engaged in the smoking of a cigarette the temperature of the subject under investigation falls considerably. In some instances a drop of six degrees centigrade has been observed while smoking. This is, of course, due purely and simply to vasoconstriction. For this reason, therefore, smoking must be completely prohibited in all forms of peripheral arterial disease. A patient who refuses to stop smoking completely and immediately is wasting both his time and that of the physician, as no successful therapeutic procedure has yet been discovered to counteract the harmful effect of smoking. It is thus not be be assumed that smoking is the direct cause of Buerger's Disease, but is rather an aggravating factor.

The vasoconstricting action of exposure to cold must also be taken into consideration in treatment. Thus in cold weather it is important that the extremities and the body be properly clothed. The use of wool undergarments, stockings and gloves is essential in cold weather. In the case of women some difficulty may be experienced in carrying out this method of dressing. In men, however, there can be no excuse for not adopting it completely. This brings up the question of change of climate for these patients. It has been my experience that they do no better in warmer climate than in one to which they are ordinarily accustomed. As a matter of fact, many patients relate that they walk and feel much better in cold weather than they do in the heat of summer.

The application of external heat to the extremities in peripheral arterial diseases is a dangerous procedure. I refer particularly to the use of hot water bottles, electric heating pads, thermostatically controlled electric cradles and various other apparatus. The great objection to these measures lies in the fact that the extremities, particularly the feet, are usually hyposensitive so that excessive heat may be applied without the patient realising 
that a severe burn is taking place. Because of this traditional and dangerous method of treatment many legs have been unnecessarily amputated because of a severe burn experienced by the application of some form of external heat. A safe and effective method of inducing vasodilatation of the legs by external. heat is the use of a hot or fairly warm sitz bath. This may be taken daily, preferably just before retiring and for a period of ten minutes or thereabouts. In no case should the temperature of the water be above $105^{\circ} \mathrm{C}$. and the level of the water should be no higher than the hips with the patient sitting in the tub.

Buerger advocated the use of a system of controlled changes in position of the legs as a means of stimulating blood flow in the lower extremities. These exercises are carried out by the patient from one to four times a day depending upon convenience or under existing circumstances. The patient lies flat on the back on a bed or couch and elevates the legs to an angle of $45^{\circ}$ or more, holding them upright in this position until blanching occurs. This usually takes about two minutes. In the next position the patient sits upright and allows the feet to hang over the edge of the bed until they become somewhat reddened. This usually takes about three minutes. After this there follows a rest period of two minutes during which time the patient is flat on the back again with the feet on the bed in a horizontal position. This cycle of three positions is repeated three or four times, depending upon circumstances. It is a good plan to follow the evening exercises immediately with the hot bath described above. After the bath the feet should be carefully dried and a fungicidal powder applied between the toes. A preparation of calcium propionate has proven most effective as a fungicide in these cases.

An excellent auxiliary vasodilating agent has been found in the use of short wave to the legs. This may be given at fifteen minute periods, usually three times a week or oftener as indicated. The current should be regulated, preferably by the patient, so that there is at no time a feeling of discomfort in the legs but rather one of moderate warmth. In administering thousands of treatments by this form of therapy I have never encountered any accidental burns or other harmful effects as one would expect from the application of external heat.

Alcohol in the form of a beverage has proved to be one of the most effective vasodilators. It may be prescribed in doses of 15 to 30 c.c, three times a day with good results. I am at present investigating a new vasodilating compound (2-Benzyl4, 5-imidazoline HCL). This compound is known as "Priscol" and is administered both by hypodermic and orally in tablet form. Results of its use in cases characterised by excessive vasospasm are so far encouraging, but a definite report cannoto be made until more experience is had with this new drug.

The introduction of various tissue extracts, such ${ }^{\complement}$ as extract of pancreas with insulin removed, various muscle extracts, kidney extract and other simila $5_{5}^{\circ}$ preparations was felt to be the answer to relief of the difficulty and disability of intermittent claudication. Prolonged experience with these various preparations usually given by hypodermic injec -0 tion, leads me to the conclusion that their value is doubtful and that the good results obtained are for $\overrightarrow{0}$ the most part psychic in nature. In order to obtain therapeutic results tremendous doses of these materials would have to be employed rathero than the small amount at present in use. Thes pain and discomfort from such large doses would of course, prohibit their practical use.

A word here concerning the use of varioust mechanical appliances to facilitate circulation in the extremities. Among these may be mentioned the "pavex" machine and various forms of inter응 mittent venous compression. It was felt for a timethat the last word had been spoken in the treat w ment of peripheral arterial diseases when these machines were introduced. Increasing experienc娄 with them, however, soon convinced the profession that the claims of their inventors were somewh to exaggerated and their practical value nil. Unfor-o tunately, in some instances, actual danger present in their use because of the trauma to thises large vessels of the thigh and the subsequento development of either gangrene or spreading in $\frac{\%}{2}$ fection or phlebothrombosis. The use of any of these machines therefore in any stage or in any variety of peripheral arterial disease is not recom- -3 mended.

Relief of vasospasm in peripheral arterial diseaseș by severing the sympathetic pathways to the ex:tremities has been recommended from many sources. The operation either in the form of a: Leriche sympathectomy or in the nature of an? extensive operation such as a lumbar or cervica $B$ ganglionectomy may produce temprary relief oß vasospasm in the extremity, but is in no sense too be considered as a cure for any of these diseases. Their effects upon the organic obstruction which is? fundamental to these conditions is nil. It is my feeling that just as effective vasodilatation can ber secured in most of the peripheral arterial diseases by the simpler and safer methods described above స్ల For this reason I do not advocate the indiscriminate application of this formidable operative procedure in peripheral arterial disease.

A word concerning the use of typhoid vaccines intravenously to induce high fever as a vasodilating aid. I have seen three cases of sudden massiveo 
gangrene of a previously healthy extremity result from typhoid vaccine intravenously as a form of treatment. It is well known that during the chill phase of the reaction there is a change in the clotting time of the blood and that extensive thrombosis and major occlusions may occur. The beneficial effects of this form of therapy are so slight in comparison to the risk attached to their use that I do not advocate this form of treatment.

The use of the various choline derivatives and vasodilators in peripheral vascular diseases has proved to be ineffective, and their use by iontophoresis is attended with considerable danger of burns.

Experience of twenty years or more with the use of intravenous injections of hypertonic saline has demonstrated the value of this form of treatment in thromboangiitis obliterans. It is not to be considered a specific for the disease nor is too much reliance to be placed upon it to the exclusion of other factors previously enumerated in the management of these cases. The only specific objective effects that have been noted with the use of intravenous saline are an immediate temporary increase in pulse amplitude in the extremities as demonstrated by the oscillometer, increased warmth of the feet, renewed toenail growth and the healing of chronic ulcers and of extensive areas of gangrene of the extremities. It is assumed, of course, that in addition to the use of saline injections the patient is not smoking and is co-operating in all other respects. The solution is administered three times a week, 300 c.c. at a time fairly rapidly by the gravity method using an eighteen or nineteen gauge needle. Five per cent sodium chloride is reserved for the younger vigorous patients, particulorly those with thromboangiitis obliterans. In the older group or in those showing a tendency to local irritation at the site of injection, three per cent sodium chloride or two per cent saline is preferable. These injections may be given in the office or at the patient's home. They are continued for many months either until improvement in symptoms is obtained or until gangrenous areas and ulcers are healed. Contraindications are hypertension, myocardial or renal disease.

The treatment of gangrene necessitates a thorough understanding of the pathogenesis of this condition. One must distinguish two forms of gangrene. One is gangrene in its pure or primary form, the type that is caused by obstruction in the vessels alone. This is encountered in its classical form in embolism or in sudden thrombosis of a large artery. Here the usual picture is sudden onset of coldness, ischemia and extreme pain in the affected limb followed by progressive blueness of the extremity ending in complete mummifica- tion or drying of the affected part. This was formerly designated "dry gangrene." The aim of treating this form of gangrene is to wait patiently for a line of demarcation to form which will indicate the ultimate extent of the gangrenous process. After this line has been established an attempt should be made to separate the dying or dead tissue from the living part. This can sometimes be accomplished by careful dissection along the line of demarcation or in some instances it may be effected by attempting to macerate the dead tissues. This is best accomplished by foot soaks of soap and water followed by the application of boric acid ointment. All efforts must be employed to avoid contamination of the gangrenous areas so that secondary infection shall not ensue. This requires careful dressings by the attending surgeon utilising all usual methods of antisepsis. In the removal of a gangrenous toe by this method, one must be particularly careful when the various tendon sheaths, particularly those of the flexor tendons of the toes are opened. The administration of penicillin intramuscularly for a few days before and after the removal of the gangrenous toe or toes may prevent the development of subsequent infection of the open tendon sheaths. Following the separation and removal of the gangrenous parts in this type, a granulating ulcer will usually be found which if not secondarily infected will usually heal by the continued application of a bland oint ment, such as boric acid. If infection of the tendon sheaths does supervene, treatment must be carried out in the manner to be described in the next paragraph.

A more common and a more dangerous form of gangrene is that seen most often in arteriosclerosis with diabetes, although it may also occur without diabetes. Here, of course, one is dealing primarily with insufficient blood supply in the extremities usually caused by arteriosclerosis obliterans. In these cases there is usually present, in greater or less degree some form of mycotic infection of the feet. These fungi are usually present in the interdigital spaces of the toes; or they may also be found in various excrescences of epithelium such as corns, calluses and other areas of the skin. These parasites may be present for many years and may cause no particular harm. In some instances, however, particularly in the interdigital spaces, they may cause maceration of the skin between the toes with resulting itching and burning. The patient is prone to tamper with these infected areas, either by rubbing the fingers between the toes or by application of a strong irritating antiseptic. If the location is in a corn or callus, the patient may attempt to remove these areas by means of infected razor blades or by picking these excrescences with dirty finger nails. In any event 
FIG. I6.-Healed supracondylar amputation.

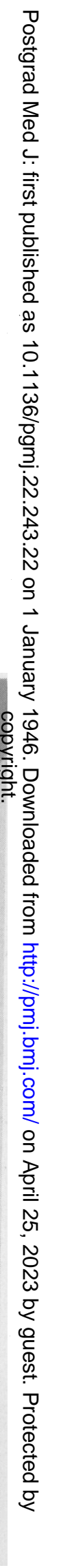


there is usually introduced a secondary pyogenic infection and from this point on real trouble develops. As a result of the secondary infection there is localised edema and extension of the infection into the adjacent tissues. This causes secondary thrombosis of the small arteries leading to the toes with the result that gangrene surely follows. This then is known as secondary or infected gangrene in contrast to that type described in the previous paragraph.

In the diabetic patient spread of the infection is more rapid and more dangerous and when the condition is not recognised and continues to spread it usually results in a purulent infection of the tendon sheaths of the foot and of the fascial spaces of the foot. On the dorsum of the foot the infection is usually not very extensive nor of very great consequence as the anatomical structures in that location are not very complex as compared to the plantar tissues. Having, therefore, the combination of gangrene and infection it is logical to assume that since the gangrene is dependent upon the development of the infection, treatment should be directed to the underlying cause of the gangrene In this case it is the infection. In other words, in secondary gangrene such as encountered in diabetics and others, one's attention should be directed not to the condition of the circulation in the extremities, but rather to the extent and treatment of the infection. This is a new approach to the treatment of diabetic gangrene and must be thoroughly understood if amputation of limbs is to be avoided. In the early stages of these infections, before pus has formed, the local application of various antiseptics will prove sufficient. Among these the most beneficial has been azochloramid in triacetin. Penicillin in high dilution and tyrothricin either in solution or in the form of a cream have also proven effective. The sulpha drugs by local application and by mouth have not proven satisfactory in secondary or infected gangrene.

When the presence of frank pus has been established in the foot immediate incision and drainage, plus the direct application of a powerful antiseptic, agent must be employed without delay. If an infected tendon sheath is found, it must be opened completely. This is also true of infected fascial spaces of the foot. These also must be opened extensively and thoroughly and the contents of infected or necrotic tissue completely removed. The resulting cavity should then be flushed out with peroxide of hydrogen followed by a wash of some mild antiseptic solution, such as Dakin's solution, zephiran or some similar compound. After this, the cavity is packed tightly with gauze, saturated either with azochloramid in triacetin or dilute penicillin or dilute tyrothricin. Of the three, I have found azochloramid preferable be- cause of its effect upon a greater number of orgañ isms than the other two agents.

A local anesthetic must never be used in makin these incisions of the feet as gangrene will certainy follow. In most cases no anaesthetic whatever is required. A sharp scissors or scalpel will usually prove most effective because these feet are for the most part hyposensitive. Where indicated, hov ever, a light general anesthetic may be used.

If toes or other parts are found to be complete gangrenous at the time of the incision the dean part may be removed safely. Dressings are changed daily, using the same routine of irrigatio?, with peroxide of hydrogen followed by a mild ant septic solution and then firm packing with satw rated gauze. It will be observed after a shogt time that as the infection is conquered, the gan. grenous process subsides. The use of penicillin systematically during this phase of the treatment of infected gangrene is still open to questiot Many cases respond favourably to the use of periig cillin systemically along with adequate local treatment of the foot, but in some cases undoubtedly due to the type of organisms present, nothing or very little can be accomplished with the use of penicillin by intramuscular injections. Therefores, too much faith should not be placed in this remedzy in the treatment of secondary gangrene.

As the infection is overcome and the gangrenot parts become demarcated and are remowed healthy granulations will appear in various pares of the foot. Persistence in this treatment will soom result in the formation of a granulating woun which will eventually epithelialise. Thus eve्c with the loss of one or more toes or even of all toes of the foot a good functioning extremity can expected.

In the gangrene of thromboangiitis obliteran one must realise that the disease is usually se limited and that if all the rules enumerated above have been carefully followed, particularly that relative to smoking, a good result can be obtainee. Here the question of infection is not very impos:tant as the condition is essentially one of primas gangrene and the object of treatment is to keep the parts as clean as possible and to allow spontaneous demarcation and separation of the gangrenows portions. Daily foot soaks of green soap anf warm water or of a mild chloramine solution afe indicated followed by the application of bland ointment such as boric acid ointment. If this too painful, the use of benzocaine or other anaes? thetic ointments may be tried.

Complete bed rest with the affected limbs in the horizontal position at all times is essential in the treatment of gangrene. In many instances the patient will apparently obtain relief from pain bج sitting upright in bed or in a chair day and niglit 
with the feet hanging. While this affords temporary relief, it also causes an extensive edema of the foot and eventually of the entire leg, which is not conducive to healing and which usually causes the gangrene and infection to spread. This condition I have designated "positional edema" and the only method of overcoming it is to insist upon the patient keeping the extremities in bed in a horizontal position at all times, after which the edema will be observed to disappear completely in about six to twelve hours.

This brings up the importance of complete bed rest in all cases of impending or threatened gangrene. An early sign of this is increasing pain in the extremities at night, whereby the patient must in some cases get off the bed and walk about the room to secure relief. Immediate hospitalisation with complete bed rest is imperative at this stage. In addition both legs are wrapped in sheet cotton from the toes to the groin to secure retention of the natural body warmth. This in itself is a powerful vasodilator and will usually give spectacular relief from night pain. Morphine and its derivatives are strictly to be avoided in the treatment of any and all phases of these diseases, as they soon become habit forming and may cause complications more difficult to treat than the original condition. The combination of no smoking, bed rest, aspirin and whiskey has proven more effective than any known narcotic.

What are the indications for amputation of a leg in peripheral arterial disease? As in other branches of medicine and surgery no hard and fast rule can be laid down. One must rather rely upon experience and good judgment based upon a thorough knowledge of the disease. In general, however, it can be stated that there are two main indications for amputation of the leg.

I. Destruction of so much of the weight bearing portion of the extremity by gangrene that a functioning leg cannot be expected.

2. Infection in the foot or leg which cannot be controlled by any of our known modern methods.

Amputation of toes before a line of demarcation has occurred is a dangerous procedure and should not be done, as it very often leads to further spread of the gangrene into the foot.

The level of amputation is important. At the outset it must be stated that indications for amputation below the knee are an adequate circulation either original or collateral below the knee and infection confined to the foot. It follows, however, that if such a combination is present, one really has no indication of amputation at all, rather one must feel that conservative therapy is in order. There are some cases, however, where the physician may feel that too much of the weight bearing portion of the foot has been destroyed and yet where circulation is apparently adequate below the knee, where this type of operation may be done. This is particularly true if the oscillometer indicates some pulsation at the ankle level.

The following technique has proven most effective for this type of operation. Three levels are marked off on the skin with the back of a scalpel on the leg to be amputated. The middle level is placed about six inches below the tibial tubercle indicating the level at which the tibia will be sawed. Another level is marked off about one inch proximal to this indicating the level of the skin incision. At this point a circular incision is made through the skin and soft parts down to the bone. No tourniquet is used and all bleeding vessels are carefully clamped as the operation proceeds. Directly over the fibula a longitudinal incision is made starting at the circular skin incision and extending proximally up to the fibula level previously marked on the skin. By blunt dissection a piece of fibula is removed using a rib cutter for the purpose corresponding to the upper and lower level marked out on the skin. After this piece of fibula has been removed, the soft parts are retracted along the tibia until the level of tibial section is reached. The periostium of the tibia is then cut and scraped distally and the bone is sawed through at that point. The sharp anterior border of the tibia is then bevelled off with either a saw or rongeur forceps and the soft parts are closed over the end of the tibia, bringing them well under the anterior surface of the exposed tibia. The skin edges are approximated carefully and no drains are used.

Most amputations of the leg are best performed above the knee where the procedure is simple, is practically shockless and has the lowest mortality rate on record for this type of operation. This is especially indicated when the oscillometer reveals no pulsation whatsoever at the ankle level of the affected limb. Without the use of a tourniquet, a circular incision is made through the skin and soft parts at the upper border of the patella down to the bone. Accurate hemostatis is essential. The soft parts are then retracted proximally for about two inches with the use of a warm wet towel. At this proximal point, the periostium of the femur is incised and scraped distally and the bone is sawed through at that level.

Here it is important to emphasise the necessity for simplicity of technique. This applies particularly to handling the sciatic nerve, which is merely cut at right angles and allowed to retract spontaneously. No injection of alcohol or other irritating manipulations are advisable. After removal of the leg, the muscles and other soft parts are 
allowed to fall together naturally and they are sewed over the end of the bone. The stump of the popliteal artery is buried. The soft parts and skin are then sewed together very carefully and no drains are used.

The question of choice of anesthesia is important. I have found that a well-administered cyclopropane anesthesia is the best in all cases, particularly in elderly people. It is accompanied with no shock whatsoever and gives no postoperative complications or discomfort. After reacting from the anesthetic, patients are allowed to sit up in bed and may eat their next regular meal if they so desire. The day after operation they are routinely taken out of bed and allowed to sit up in a chair for as long as they please and the same for every day thereafter. On the third or fourth day they may be allowed to get about on crutches if they desire. On the sixth or seventh day stitches may be removed and if primary union is obtained, as it usually is, they are sent home on the eighth or the tenth day completely healed. An artificial leg may be ordered immediately and may be applied as soon as ready by the limb maker. With a mortality rate of approximately five per cent for this type of operation it is obvious that there is no particular advantage in the use of refrigeration as a method of anesthesia in these amputations. It is the simplicity and gentleness of the operative technique that is important rather than the type of anesthesia that is used, although I do feel that cyclopropane is highly preferable to refrigeration.

\section{Summary}

In the field of peripheral arterial diseases it is essential that the doctor know how to diagnose a case in its earliest stages. The method of doing this has been described. After making a proper diagnosis, the question of management of the case is then in order. Fundamental principles based upon long experience in this field have been described, enabling the doctor to treat these patients in an intelligent manner. Gangrene is, for the most part, a preventable disease and a good deal of the responsibility for this prevention rests upon intelligence and well-founded principles of treatment rather than upon the employment of some new gadget or apparatus or single method of treatment which has received unwarranted popularity. Amputation of limbs, when necessary, must be done in a simple and gentle manner for the ultimate aim of saving the patient's life and of getting the patient back to an ambulatory condition as soon as possible.

\section{MEDICAL, NEWS}

There will be a clinical meeting of the Medical Society of the L.C.C. Service on Thursday, February 7 th, at 2.45 p.m., at MAUDSLEy Hospital, Denmark Hill, S.E.5, when members of the staff of Maudsley Hospital will demonstrate cases. 